\title{
Methods in angle-resolved photoelectron diffraction: Slab method versus separable propagator cluster approach
}

\author{
Huasheng Wu, C. Y. Ng, T. P. Chu, and S. Y. Tong \\ Department of Physics, The University of Hong Kong, Hong Kong, China
}

(Received 25 July 1997; revised manuscript received 12 January 1998)

\begin{abstract}
We have compared multiple-scattering results of angle-resolved photoelectron diffraction spectra between the exact slab method and the separable propagator perturbation cluster method. In the slab method, the source wave and multiple scattering within strongly scattering layers are expanded in spherical waves while the scattering among different layers is expressed in plane waves. The transformation between spherical waves and plane waves is done exactly. The plane waves are then matched across the solid-vacuum interface to a single outgoing plane wave in the detector's direction. The slab is infinitely extended parallel to the surface. Normal to the surface, enough layers are included to ensure convergence of the calculated intensity. The separable propagator perturbation approach uses two approximations: (i) A separable representation of the Green'sfunction propagator and (ii) a perturbation expansion of multiple-scattering terms. The cluster size is finite, typically containing 50 atoms or less. Results of this study show that using a cluster of 148 atoms, the largest cluster used to date, the cluster size is still too small for the cluster results on $\mathrm{Ni}(001)$ to converge with those of the slab method. Ideas to improve the perturbation expansion cluster method are discussed.
\end{abstract}

[S0163-1829(98)01324-1]

\section{INTRODUCTION}

Because of its chemical specificity, angle-resolved photoelectron diffraction is very useful in studying the geometric structure, electronic properties, and magnetic properties of clean and adsorbate-covered surfaces. For clean surfaces, high-resolution measurements can separate out the photoelectron signal from surface-induced shifted core levels. The technique is a local probe that does not require long-range order [as opposed to low-energy electron diffraction (LEED) $\left.)^{1,2}\right]$; however, the systems studied in detail so far all possess ordered two-dimensional periodicity, at least in the crystal structure of the substrate. This is because in order to gather enough signal for the measurement, multiple equivalent events must be cumulated.

To describe the experiment for systems with twodimensional periodicity, it is convenient to use a slab geometry to track the emission and diffraction process of the photoelectron. The experimental situation is to measure the flux of an outgoing plane wave in a given direction $\mathbf{k}_{f}$ in the far-field region (i.e., $d w / d \Omega_{\mathbf{k}_{f}}$, defined as the number of electrons entering solid angle $d \Omega_{\mathbf{k}_{f}}$ per unit time). A proper description requires matching of the electron wave field across the solid-vacuum interface. In the slab approach, ${ }^{3,4}$ this matching is done by requiring the conservation of parallel momentum $\mathbf{k}_{f \|}^{\text {in }}=\mathbf{k}_{f \|}^{\text {out }}$ inside and outside the solid. In the slab method, the photoelectron excitation matrix elements are explicitly calculated and multiple scattering within strongly scattering "layers" is carried out in the sphericalwave representation. Multiple scattering among layers in which the scattering is less strong is carried out in the planewave representation. The transformation between spherical and plane-wave representations is done by well-established procedures. ${ }^{5}$ Similar to the combined-space method ${ }^{6}$ developed for LEED, a strongly scattering "layer'" could mean a single plane of atoms or a slab containing many planes of closely spaced atoms. The slab method has been used to determine the surface geometry of adsorbed atoms and molecules on different substrates. ${ }^{7-17}$ Recently, the slab method has been extended to include relativistic magnetic-spin dichroism effects. ${ }^{18-22}$ The slab method of angle-resolved photoelectron diffraction shares many features with the slab approach of LEED, such as the use of symmetry, calculating layer emission, and diffraction matrices once and using these for multiple variations of interlayer distances, using $R$ factors for structural determination, etc. ${ }^{1,2}$

Recently, Rehr and Albers ${ }^{23}$ introduced a separable propagator perturbation cluster approach. In this method, the multiple scattering is divided into scattering paths in a perturbation expansion, with each order indicating the number of times a photoelectron is scattered by an ion-core potential. Because two-dimensional periodicity is not assumed, the atoms are confined to a finite cluster. ${ }^{24,25}$ To go beyond thirdorder perturbation expansion, it becomes necessary to introduce a separable representation for the Green's function propagator. While the separable expansion is formally exact if taken to infinite order, most calculations included only $(6 \times 6)$ matrices in the separable form. Rher and Albers have shown that this approximation method converges for $\mathrm{X}$-ray adsorption fine structure ${ }^{23}$ (XAFS). Recently, a number of authors have applied this method to angle-resolved photoelectron diffraction (ARPD) spectroscopy. ${ }^{26}$

The purpose of this paper is to study the convergence of the Rehr and Albers (RA) method when applied to ARPD. Immediately, we note a number of differences between XAFS and ARPD: (i) The lowest order, i.e., strongest event, in the scattered wave of XAFS involves at least two backscatterings, once at a neighboring atom and once at the emitting atom. By contrast, the strongest scattered wave in ARPD may not contain any backscattering at all. There are many 
such strongly scattered amplitudes in glancing angle ARPD. A backscattering event is defined as one that has a scattering angle larger than $90^{\circ}$. (ii) In XAFS, an angular integral of the interference is taken at the emitting site. The $4 \pi$ integral smooths out angular anisotropies. No such angular integral is taken in ARPD. Thus, we expect that ARPD would require a better convergence from an approximation method.

We test the RA method by choosing a variety of systems to test the different approximations used in the method. We use horizontal linear chains of atoms of different lengths to test the multiple-scattering perturbation expansion. We use a vertical chain with an emitter at one end to test the separable approximation. We use a cluster of $148 \mathrm{Ni}$ atoms arranged in a $\mathrm{Ni}(001)$ lattice to test the convergence of cluster size. The major finding of this paper is that even using a cluster of 148 atoms, the cluster results still have not converged to that of the slab method. Ideas to improve the perturbation expansion cluster approach are discussed.

The remainder of this paper is organized as follows: Section II summarizes the main features of the slab method. Section III does the same for the RA method. The perturbation expansion convergence test using horizontal chains of atoms is presented in Sec. IV. In Sec. V, we test the (6 $\times 6)$ separable form. We test results of a 148-atom $\mathrm{Ni}(001)$ cluster with the slab method in Sec. VI. The error in the boundary condition used in the RA method is examined in Sec. VII. In Sec. VIII, we present conclusions and comments.

\section{THE SLAB METHOD}

In the slab method, we divide a system with twodimensional periodicity into atomic planes or composite layers parallel to the surface. Each plane or composite layer must have a two-dimensional periodicity commensurate with the other planes/layers of the system. No order is required in the stacking sequence or interlayer spacing normal to the surface. The number of layers included in the calculation is determined by the attenuation of the electron inside the material. Typically, the calculation seeks intensity convergence of $\sim 2 \%$, meaning that 20-40 layers are included for energies up to $400 \mathrm{eV}$, depending on the inelastic damping.

The detection direction defines a parallel wave vector $\mathbf{k}_{f \|}$. On the vacuum side, a real wave vector is defined by

$$
\mathbf{k}_{f}^{\text {out }}=\left(\mathbf{k}_{f \|}, k_{\perp}^{\text {out }} \hat{e}_{z}\right),
$$

where $k_{\perp}^{\text {out }}=\sqrt{\left(2 m / \hbar^{2}\right) E-k_{f \|}^{2}}$ and $\hat{e}_{z}$ is a unit vector pointing normally from solid to vacuum. The kinetic energy of the photoelectron is $E$. Inside the material and in the interstitial region outside the muffin-tin spheres, the wave vector is complex and is given by

$$
\mathbf{k}_{\mathrm{in}}^{ \pm}=\left(\mathbf{k}_{f \|}, \pm k_{\perp \text { in }} \hat{e}_{z}\right)
$$

where

$$
k_{\perp \text { in }}=\sqrt{\left(2 m / \hbar^{2}\right)\left(E+V_{0}+i V_{I}\right)-\mathbf{k}_{f \|}^{2}} .
$$

In Eq. (3), $V_{0}$ is the inner potential and $V_{I}$ is the imaginary potential inside the solid.

Diffraction of a photoelectron by an ordered lattice plane changes its parallel momentum by a two-dimensional recip- rocal lattice vector $\mathbf{g}$; hence, it is necessary to consider additional plane-wave directions given by

$$
\mathbf{k}_{\mathrm{in}}^{ \pm}(\mathbf{g})=\left[\mathbf{k}_{f \|}+\mathbf{g}, \pm k_{\perp \text { in }}(\mathbf{g}) \hat{e}_{z}\right]
$$

where

$$
k_{\perp \text { in }}(\mathbf{g})=\sqrt{\left(2 m / \hbar^{2}\right)\left(E+V_{0}+i V_{I}\right)-\left(\mathbf{k}_{f \|}+\mathbf{g}\right)^{2}} .
$$

A photoelectron in any $\mathbf{k}_{f \|}+\mathbf{g}$ direction can be scattered into the detector's direction by a lattice plane. The photoelectron wave field in the interstitial region is given by a linear combination of plane waves with wave vectors given in Eq. (4). In the plane-wave representation, reflection and transmission matrices $R_{\mathbf{g}^{\prime}, \mathbf{g}}^{ \pm \pm}, T_{\mathbf{g}^{\prime} \mathbf{g}}^{ \pm \pm}$from individual planes or composite layers are generated using the method of LEED. ${ }^{1,2,27}$ The only additional quantities to add are the source layer matrices for the photoelectron. These are given $b^{3}$

$$
\begin{aligned}
A\left[\mathbf{k}_{\mathrm{in}}^{ \pm}(\mathbf{g})\right]= & \alpha_{0} \sum_{\beta} \sum_{L L_{1} L_{2}} \frac{Y_{L_{1}}\left[k_{\mathrm{in}}^{ \pm}(\mathbf{g})\right]\left(1-\tau^{\beta} \hat{G}^{\beta \alpha}\right)_{L L_{1}}^{-1}}{k_{\perp \text { in }}(\mathbf{g})} \\
& \times\left(1-t \hat{G}^{\alpha \alpha}\right)_{L_{1} L_{2}}^{-1} M_{L_{2}}^{\alpha} e^{-i \mathbf{k}_{\mathrm{in}}^{ \pm}(\mathbf{g}) \cdot \mathbf{d}_{\beta} .}
\end{aligned}
$$

Notice that the source wave is evaluated not only in the $\mathbf{k}_{f \|}$ direction but in all the $\mathbf{k}_{f \|}+\mathbf{g}$ directions. The constant in Eq. (6) is

$$
\alpha_{0}=\left(\frac{2 m}{\hbar^{2}}\right)\left(\frac{2 i \pi}{N A}\right),
$$

where $A$ is the two-dimensional unit cell area in the layer. The vector $M_{L}^{\alpha}$ contains the photoelectron excitation elements. It is given by ${ }^{4}$

$$
\begin{aligned}
M_{L}^{\alpha}= & D \mathcal{A} \cdot \int d \Omega_{\alpha} Y_{L}^{*}\left(\hat{r}_{\alpha}\right) \hat{r}_{\alpha} Y_{L_{1}}\left(\hat{r}_{\alpha}\right) \\
& \times \int r_{\alpha}^{2} d r_{\alpha} e^{i \delta_{l}^{\alpha}} R_{l}^{f}\left(r_{\alpha}\right) \frac{d V}{d r_{\alpha}} R_{l_{i}}^{\alpha}\left(r_{\alpha}\right),
\end{aligned}
$$

where $\mathcal{A}$ is the photon's vector potential, $D=$ $(-1)^{l} i^{l+1}(e \hbar / m c)\left[1 /\left(E-\varepsilon_{i}\right)\right]$, with $\varepsilon_{i}=$ the energy of the core level and $\mathbf{r}_{\alpha}$ has its origin at the nucleus of the emitting atom. The quantity $\tau_{L L}^{\beta}$ in Eq. (6) is the in-plane multiplescattering matrix for a Bravais lattice. The sum $\beta$ is over all Bravais lattices, which make up a composite layer. The layer propagators $\hat{G}^{\beta \alpha}$ are defined in Eq. (42) of Ref. 2. The emitting atom can be situated in any layer of the slab and the photoelectron intensity is the sum of independent events. Applying the $\mathbf{k}_{f \|}$ conservation law across the interface, and neglecting multiple reflections at the inner potential step, the differential flux in the $\mathbf{k}_{f}$ direction is given by ${ }^{5}$

$$
\frac{d \omega}{d \Omega_{\hat{k}_{f}}}=\frac{\hbar}{m} \frac{A^{2}}{(2 \pi)^{2}}\left(k_{\perp}^{\text {out }}\right)^{2} k_{f}^{\text {out }}\left|C\left(\mathbf{k}_{\text {in }}^{+}\right)\right|^{2} .
$$

Here, $C\left(\mathbf{k}_{\text {in }}^{+}\right)$is the plane-wave coefficient just inside the solid. This coefficient is calculated by starting with the layer source coefficients $A_{\alpha}\left[\mathbf{k}_{\text {in }}^{ \pm}(\mathbf{g})\right]$ and applying the standard LEED layer stacking methods such as the combined-space method, ${ }^{6}$ layer doubling, or renormalized forward 


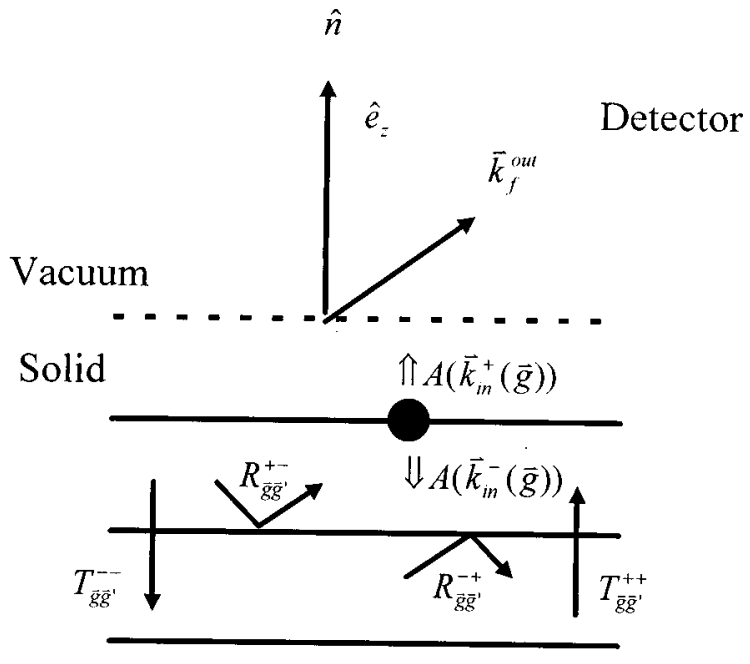

FIG. 1. Schematic diagram of slab method showing the source vectors $A\left[\mathbf{k}_{\mathrm{in}}^{ \pm}(\mathbf{g})\right]$ and layer scattering matrices $T_{\mathbf{g g}^{\prime}}^{ \pm \pm}$, and $R_{\mathbf{g g}^{\prime}}^{ \pm \pm}$.

scattering ${ }^{1,2}$ method to obtain converged plane-wave coefficients at the interface (see Fig. 1). Thus, the slab method of photoelectron diffraction differs from LEED theory only in that instead of considering a single incoming plane wave, there are now two sets of plane waves $\mathbf{k}_{\text {in }}^{ \pm}(\mathbf{g})$ with coefficients $A\left[\mathbf{k}_{\mathrm{in}}^{ \pm}(\mathbf{g})\right]$. These plane waves are created at a given atom in a given layer and their diffracted amplitudes are summed coherently. For each detection direction $\mathbf{k}_{f}^{\text {out }}$, the slab method produces simultaneously the differential fluxes for all the related $\mathbf{k}_{f \|}+\mathbf{g}$ directions. In terms of summing multiple-scattering paths within a slab, the slab method of ARPD is exact, just as LEED theory is exact in this respect. Approximations in the dynamical model such as the muffintin potential, the Debye-Waller model for temperature effects, the no-reflection condition at the inner potential step, etc., introduce uncertainties in the theory. However, these factors affect equally the slab and cluster approaches. Isotropic and anisotropic temperature corrections have been introduced in the slab method. ${ }^{28-30}$ The angular dependence of the Debye-Waller factor is accounted for as a cosine function in the cluster approach, while in the slab method, an expansion in partial waves is used. Because of the differences in the treatment of the Debye-Waller factor, all the tests done in this work are for the case of no temperature correction. Temperature correction, of course, provides an additional damping effect in real systems and can aid significantly in the convergence of the perturbation expansion. Thus, the results of this paper can be viewed as setting the worst-case limit for the convergence of the perturbation expansion under the quoted electron mean free path.

\section{THE SEPARABLE PROPAGATOR PERTURBATION METHOD}

In the Rehr-Alber cluster approach, ${ }^{23}$ the spherical wave representation of the electron propagator $G_{L, L^{\prime}}\left[k\left(\mathbf{R}-\mathbf{R}^{\prime}\right)\right]$ is expressed as a separable sum:

$$
G_{L, L^{\prime}}(\boldsymbol{\rho})=\frac{e^{i \rho}}{\rho} \sum_{\lambda=0}^{\omega} \tilde{\Gamma}_{\lambda}^{L}(\boldsymbol{\rho}) \Gamma_{\lambda}^{L^{\prime}}(\boldsymbol{\rho}),
$$

where $\boldsymbol{\rho}=k\left(\mathbf{R}-\mathbf{R}^{\prime}\right)$. The value of $\omega$ depends on $L$ and $L^{\prime}$. This transformation is done in two steps. The first step is to separate $G_{L, L^{\prime}}(\boldsymbol{\rho})$ into energy-dependent radial and angulardependent terms by rotating $\hat{\rho}$ onto the $z$ axis of the coordinate system. This leads to the following expansion for $G_{L, L^{\prime}}(\boldsymbol{\rho})$ :

$$
G_{L, L^{\prime}}(\boldsymbol{\rho})=\frac{e^{i \rho}}{\rho} \sum_{\mu=-l}^{l} R_{m \mu}^{l}\left(\hat{\rho}^{-1}\right) g_{l l}^{(|\mu|)}(\rho) R_{\mu m^{\prime}}^{l^{\prime}}(\hat{\rho}) .
$$

In Eq. (10), $R_{m \mu}^{l}(\hat{\rho})$ rotates $\hat{\rho}$ onto the $z$ axis. The second step involves a contour integration that separates the indices $l$ and $l^{\prime}$ in $g_{l l^{\prime}}^{(|\mu|)}(\rho)$. The resulting expression is

$$
g_{l l^{\prime}}^{(|\mu|)}(\rho)=\sum_{\nu=0}^{\min \left[l, l^{\prime}-|\mu|\right]} \tilde{\gamma}_{\mu \nu}^{l}(\rho) \gamma_{\mu \nu}^{l^{\prime}}(\rho) \quad(\mu \geqslant 0),
$$

where the spherical coefficient $\gamma_{\mu \nu}^{l}(\rho)$ and $\tilde{\gamma}_{\mu \nu}^{l}(\rho)$ are given by

$$
\gamma_{\mu \nu}^{l}(\rho)=\frac{(-1)^{\mu} N_{l \mu} C_{l}^{(\mu+\nu)}(z) z^{\mu+\nu}}{(\mu+\nu) !}
$$

and

$$
\tilde{\gamma}_{\mu \nu}^{l}(\rho)=\frac{(2 l+1) C_{l}^{(\nu)}(z) z^{\nu}}{\left(N_{l \mu} \nu !\right)} .
$$

In Eqs. (12) and (13), $z=1 / i \rho$ and $C_{l}^{(\nu)}(z)$ $=\left(d^{\nu} / d z^{\nu}\right) C_{l}(z)$, with $C_{l}(z)$ being the $l$ th degree polynomial factor of the spherical Hankel function.

So far, the transformation is formally exact as long as enough basis waves are included. The first approximation in this method is to evaluate multiple-scattering paths by perturbation expansion and to stop the expansion at a finite order. The $N$ th path, with $N-1$ scatterers for the total propagator corresponding to Fig. 2 is

$$
\begin{aligned}
G_{L_{N}, L_{0}}^{(N-1)}\left(\mathbf{R}_{1}, \ldots, \mathbf{R}_{N}\right)= & \sum_{\left(L_{l}\right)} G_{L_{N}, L_{N-1}}\left(\boldsymbol{\rho}_{N}\right) \cdots t_{l_{2}}\left(\mathbf{R}_{2}\right) \\
& \times G_{L_{2}, L_{1}}\left(\boldsymbol{\rho}_{2}\right) t_{l_{2}}\left(\mathbf{R}_{1}\right) G_{L_{1}, L_{0}}\left(\boldsymbol{\rho}_{1}\right)
\end{aligned}
$$

and substituting from Eq. (9), we obtain

$$
\begin{aligned}
G_{L_{N}, L_{0}}^{(N-1)}\left(\mathbf{R}_{1}, \ldots, \mathbf{R}_{N}\right)= & \frac{e^{\left(\rho_{1}+\rho_{2}+\cdots+\rho_{N}\right)}}{\rho_{1} \rho_{2} \ldots \rho_{N}} \sum_{\left\{\lambda_{i}\right\}} M_{\lambda_{1}, \lambda_{N}}^{L_{0}, L_{N}}\left(\boldsymbol{\rho}_{1}, \boldsymbol{\rho}_{N}\right) \\
& \times F_{\lambda_{N}, \lambda_{N-1}}\left(\boldsymbol{\rho}_{N}, \boldsymbol{\rho}_{N-1}\right) \times \cdots \\
& \times F_{\lambda_{3}, \lambda_{2}}\left(\boldsymbol{\rho}_{3}, \boldsymbol{\rho}_{2}\right) F_{\lambda_{2}, \lambda_{1}}\left(\boldsymbol{\rho}_{2}, \boldsymbol{\rho}_{1}\right),
\end{aligned}
$$

where the separable scattering-amplitude matrices $F_{\lambda \lambda^{\prime}}\left(\boldsymbol{\rho \boldsymbol { \rho } ^ { \prime }}\right)$ at each site are given by

$$
F_{\lambda \lambda^{\prime}}\left(\boldsymbol{\rho}, \boldsymbol{\rho}^{\prime}\right)=\sum_{L} t_{l} \Gamma_{\lambda}^{L}(\boldsymbol{\rho}) \widetilde{\Gamma}_{\lambda^{\prime}}^{L}\left(\boldsymbol{\rho}^{\prime}\right)
$$




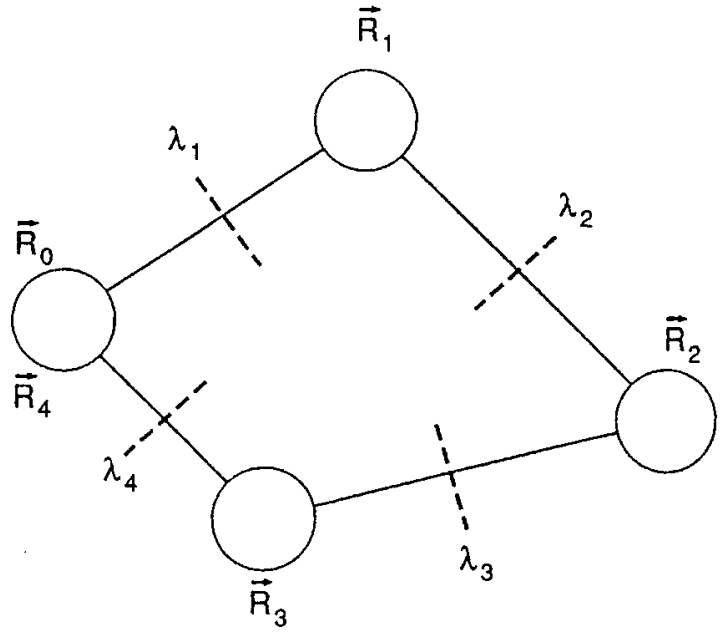

FIG. 2. Four-leg scattering path with each solid line representing a two-body Green's-function propagator and each circle (except for $\mathbf{R}_{0}$ and $\mathbf{R}_{4}$ ) a scattering center. The dashed lines represent the separation of each propagator into sums over $\lambda_{i}$ (after Ref. 23).

and the angular-momentum-dependent termination matrix is

$$
M_{\lambda_{0}, \lambda_{N}}^{L_{0}, L_{N}}\left(\boldsymbol{\rho}_{1}, \boldsymbol{\rho}_{N}\right)=\Gamma_{\lambda_{0}}^{L_{0}}\left(\boldsymbol{\rho}_{1}\right) \widetilde{\Gamma}_{\lambda_{N}}^{L_{N}}\left(\boldsymbol{\rho}_{N}\right) .
$$

In $\quad$ Eq. $\quad(16), \quad \Gamma_{\lambda}^{L}(\boldsymbol{\rho})=R_{\mu m}^{l}\left(\Omega_{\hat{\rho}}\right) \gamma_{\mu \nu}^{l}(\rho) \quad$ and $\quad \widetilde{\Gamma}_{\lambda}^{L}(\boldsymbol{\rho})$ $=R_{m \mu}^{l}\left(\Omega_{\hat{\rho}}^{-1}\right) \tilde{\gamma}_{\mu \nu}^{l}(\rho)$, where $R_{\mu m}^{l}\left(\Omega_{\hat{\rho}}\right)$ is a rotation matrix that rotates the bond direction $\hat{\rho}$ onto the $\hat{z}$ axis of the coordinate system. The spherical expansion coefficients $\gamma_{\mu \nu}^{l}(\rho)$ and $\tilde{\gamma}_{\mu \nu}^{l}(\rho)$ are given in Eq. (12) of Ref. 23.

It is important to realize that for strongly scattering paths, the perturbation expansion may diverge. If the perturbation sum fails to converge, the method will give the wrong result no matter how many orders are included. In fact, when divergence occurs for a particular scattering pathway, the calculated amplitude increases rapidly as higher orders are added. If the perturbation order is artificially limited, then a divergent amplitude may appear physical, but the value is wrong. Therefore, to ensure that divergent terms are properly flushed out, it is important to increase the multiple scattering (MS) order [i.e., the largest number of $t$ matrices included in Eq. (14)] to see if the calculated intensity converges or not. For general systems with no long-range order, conventional evaluation calculations have restricted the cluster size to less than 50 atoms and for such a cluster size, the MS order is restricted to three or four. Recently, Wu et al. ${ }^{31}$ have introduced a backward summing method, which is very efficient in summing the perturbation expansion. This method calculates the amplitudes $A_{i j}^{(m)}$, which represent $m$ th-order scatterings in which an electron starts at atom $i$ and reaches atom $j$, followed by all subsequent scatterings until it is collected at the detector. The indices $i, j$ run over all $i \neq j$ combinations of atoms in a cluster. The calculation starts with the last scattering amplitudes $A_{i j}^{(m)}$, where $j$ is the atom of the last scattering before the electron is collected at the detector. The method then traces backwards all scattering paths towards the emitter. The computation time depends linearly on the MS order. All cluster results in this paper are done using the backward summing method. We increase the MS order in each calculation until the intensity converges, if that happens. In cases where the intensity diverges, the MS order is stopped at a high number.

The second approximation of the RA method is to keep only a few terms in the $\mu, \nu$ indices. Typical calculations kept $(\mu, \nu)=(0,0),( \pm 1,0),(0,1)$, and $( \pm 2,0)$, i.e., $(6 \times 6)$ matrices. In Sec. V, we shall examine whether this is enough in ARPD for typical systems of interest.

\section{THE MULTIPLE-SCATTERING EXPANSION APPROXIMATION}

The RA method sums multiple-scattering terms by perturbation expansion and cuts off at a finite order. We can learn from an analogy in LEED, which has to deal with similar scattering terms. In LEED, the multiple scattering within a plane of atoms is grouped into a layer matrix of the form [see Eq. (58) of Ref. 27]:

$$
\tau_{L L^{\prime}}=t_{l}\left[\underset{\approx}{1}-{\underset{\sim}{G}}_{\sim}^{S P} \underset{\sim}{t}\right]_{L L^{\prime}}^{-1},
$$

where $\tau_{L L}$, is a layer scattering matrix, $t_{l}$ is an atomic scattering matrix (vector), and $G_{L L^{\prime}}^{S P}$ is the sum of all two-body propagators in that layer. This sum is given by ${ }^{27}$

$$
G_{L L^{\prime}}^{S P}=\sum_{\mathbf{P} \neq 0}^{\infty} G_{L L^{\prime}}(\mathbf{P}) e^{-i \mathbf{k} \cdot \mathbf{P}},
$$

where the vector $\boldsymbol{P}$ connects all pairs of atoms separated by distance $|\boldsymbol{P}|$ in the layer. If one uses the perturbation expansion, one can write in terms of two-body propagators by expanding Eqs. (18) and (19) into

$$
\tau_{L L}=t_{l}+t_{l} G_{L L^{\prime}}^{S P} t_{l^{\prime}}+t_{l} G_{L L_{1}}^{S P} t_{l_{1}} G_{L_{1} L^{\prime}}^{S P} t_{l^{\prime}}+\cdots
$$

The question is: Do Eqs. (18) and (20) always produce the same results?

Mathematically, the expansion in Eq. (20) is valid and the two equations will produce the same results if and only if the largest eigenvalue of $G_{L L^{\prime}}^{S P} t_{l^{\prime}}$ has a magnitude less than unity. Otherwise, the perturbation expansion in Eq. (20) will diverge to unphysical results, while the matrix inversion in Eq. (18) will remain valid. Since $G_{L L^{\prime}}^{S P} t_{l^{\prime}}$ is complex, the

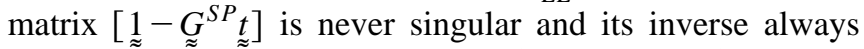
exists. The slab method of ARPD (Refs. 3 and 4) uses the matrix inversion form of Eq. (18) to treat multiple scatterings in a layer.

If only a few atoms in a chain or cluster are included in a RA calculation, then the results will always converge with a high enough MS order. This is because a short chain has only a few strong forward scattering paths and after these are exhausted, higher orders belong to backscattering events, which rapidly converge. In real situations, the system under measurement is macroscopic, often involving the scattering from hundreds of atoms. The multiple scattering is, of course, limited by inelastic damping. To achieve true convergence, it is necessary to keep increasing the number of atoms until additional forward scattering paths do not appreciably change the total intensity. It is also necessary to make sure that the MS order is large enough. 
Horizontal Ni Chain
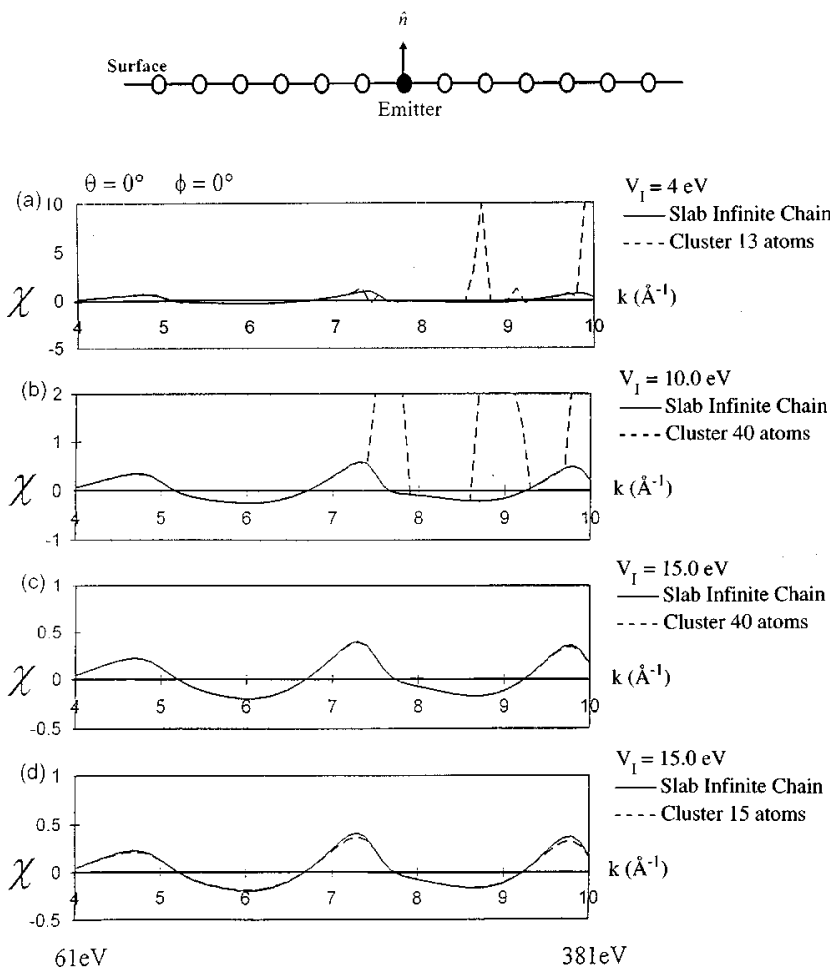

FIG. 3. (a) The RA result for a 13-atom chain, $2.49 \AA$ apart (broken line), compared to the slab result for an infinite chain (solid line). The RA result shows divergence. (b) The RA result (broken line) for a 40-atom chain. The result shows divergence. The slab result for an infinite chain is the solid line. (c) and (d) The RA result (broken line) for 40-atom and 15-atom chains, respectively, showing convergence to the infinite chain slab result (solid line).

We show the perturbation expansion test results in Fig. 3 (a) for a horizontal linear chain of $13 \mathrm{Ni}$ atoms. The detection direction is normal to the chain. The emitting atom is at the center of the chain and the atoms are placed at the $\mathrm{Ni}-\mathrm{Ni}$ nearest-neighbor distance of $2.49 \AA$. The inelastic damping is $V_{I}=4.0 \mathrm{eV}$, a number usually used in LEED calculations at these energies and no temperature correction is included. The figure shows that the normalized intensity, defined as $\left(I-I_{0}\right) / I_{0}$ where $I$ is the total intensity and $I_{0}$ is the unscattered (direct) intensity, calculated by the RA method via the backward summing scheme diverges to unphysical results at a number of wave numbers (broken line). The normalized intensity for an infinite horizontal Ni chain, calculated by the slab method, is also shown (solid line). Figure 3(b) shows the RA result for a 40-atom horizontal Ni chain. The damping is increased to $V_{I}=10 \mathrm{eV}$. All the atoms in the chain are emitters and the sum of the normalized intensities is shown (broken line). We see that even at this very large damping, the perturbation expansion is still divergent, producing unphysical intensities at a number of wave numbers. In the figure, we also show the intensity from the slab calculation for an infinitely extended horizontal Ni chain (solid line). Figure 3(c) shows the cluster result (40 atoms, all atoms are emitters) with $V_{I}=15 \mathrm{eV}$. At this damping, the RA intensity finally converges to that of the slab (infinite chain) result. Figure 3(d) shows the cluster result with 15 atoms. The good agreement with the slab result shows that with $V_{I}=15 \mathrm{eV}$, a
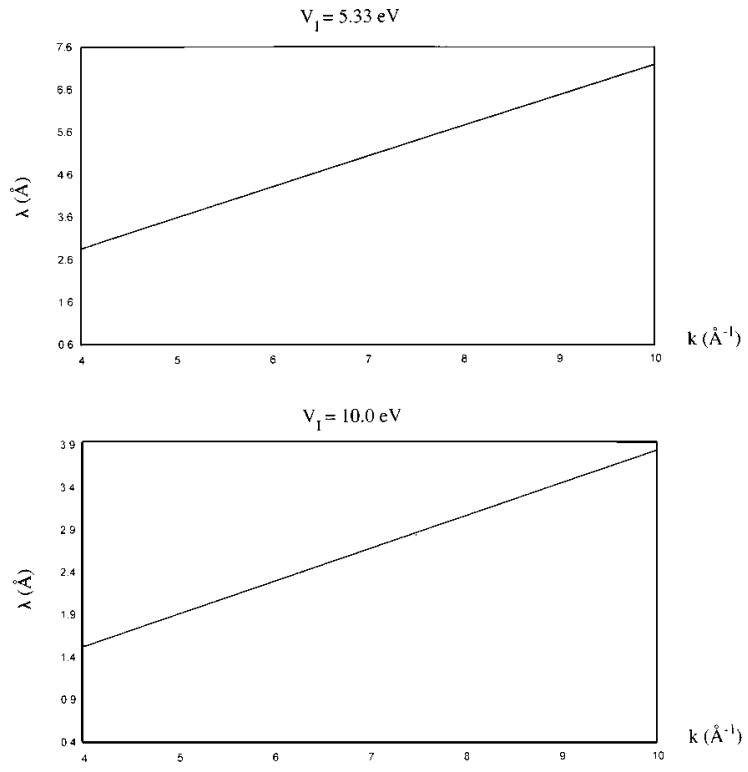

FIG. 4. Plot of the mean free path vs wave number for $V_{I}$ $=5.33$ and $10 \mathrm{eV}$.

linear chain of 15 atoms is already quite convergent. In an infinitely long chain, all atoms are equivalent. The results of a single atom emitting and all atoms emitting should be identical, except for a factor of $n$, the number of atoms in the chain. The fact that in Figs. 3(c) and 3(d), the cluster results with all atoms emitting converge to the slab results shows that for a 15 - or 40 -atom chain with $15-\mathrm{eV}$ damping, the edge effect is small.

We point out that in order to obtain the good agreement shown in Figs. 3(c) and 3(d), we have used complex $k$ in Eq. (15), wherever appropriate. Previous calculations ${ }^{26}$ have used the complex $k$ only in the exponential function $e^{i k R}$ but the real part of $k$ is used elsewhere in Eq. (15).

In this section, we have established that for linear $\mathrm{Ni}$ chains with 13-40 atoms, the backward summing scheme of the MS expansion diverges with typical dampings used in LEED calculations. With very large dampings, the cluster linear chain result can be brought to convergence. In Figs. 4 and 5, we show the relation between the electron mean free path $\lambda$ vs wave number for various choices of damping. The electron mean free path is defined as the distance in a solid at which the initial intensity has decreased by $e^{-1}$. For the majority of materials, actual mean free paths in solids correspond to damping values between 3.5 to $5.5 \mathrm{eV}$. These values are used in LEED calculations.

\section{CONVERGENCE TEST FOR THE SEPARABLE REPRESENTATION}

In the RA method, the two-particle propagator $G_{L L^{\prime}}\left(k\left|\mathbf{R}-\mathbf{R}^{\prime}\right|\right)$ is expressed as a separable sum [Eq. (9)]. In actual applications, the order included is usually $(6 \times 6)$, which is correct to terms $O\left(1 /(k R)^{2}\right)$. In this section, we investigate errors caused by the finite order in the separable form. We show in Fig. 6 the slab vs cluster calculations of normalized intensities for a vertical four-atom $\mathrm{Ni}$ chain separated by $2.49 \AA$. The MS order included is 20 . With this high MS order for the short chain, all forward scattering events in 

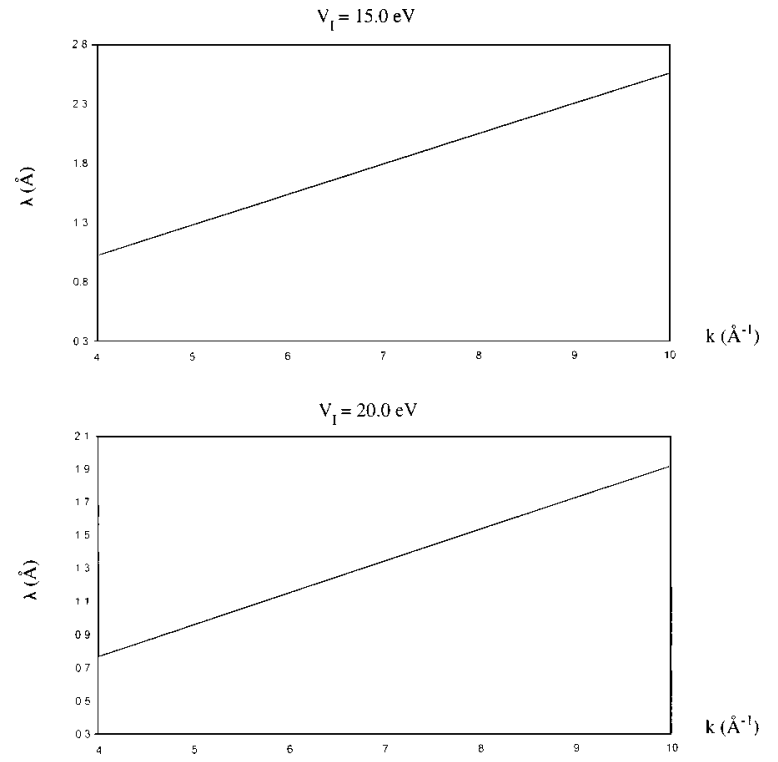

FIG. 5. Plot of the mean free path vs wave number for $V_{I}=15$ and $20 \mathrm{eV}$.

the chain would have been included and the only high-order events discarded in the RA method are those that contain many backscatterings. Since high-order backscatterings are small, their discard in this case is certainly justified. Therefore, any difference between the RA calculation and the slab calculation for this normal emission four-atom chain with

\section{Vertical 4-atom Chain}
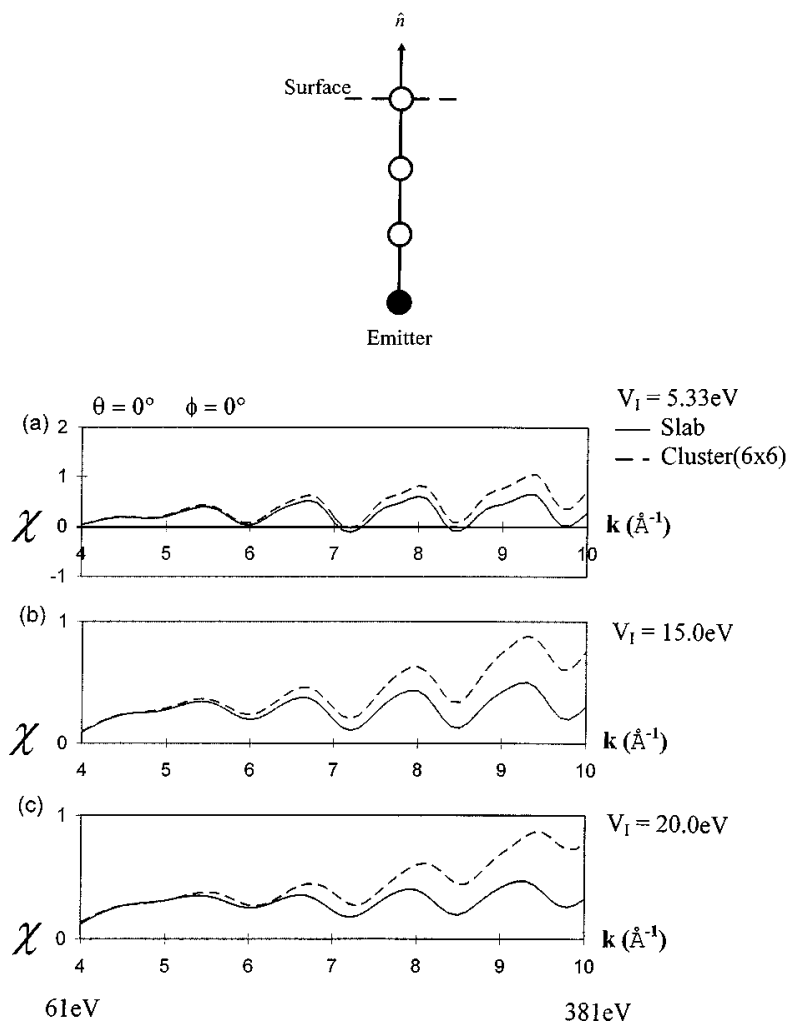

FIG. 6. The slab result (solid line) vs the RA cluster result (dotted line) for a four-atom vertical $\mathrm{Ni}$ chain, using $(6 \times 6)$ matrices in the propagator separable representation.

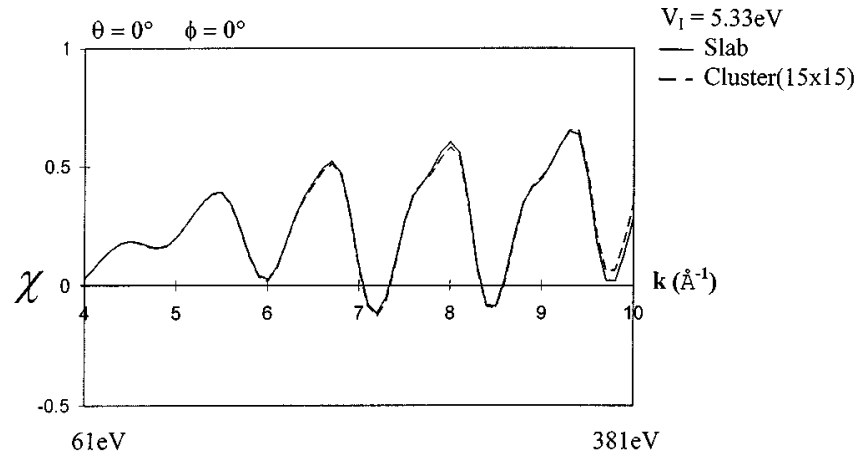

FIG. 7. Same as in Fig. 6, except $(15 \times 15)$ matrices are used in the RA method.

$\mathrm{MS}=20$ must be due to the separable representation truncation.

In Figs. 6(a)-6(c), we show the comparison for this system with $V_{I}=5,15$, and $20 \mathrm{eV}$, respectively. The emitter is placed at the deep end of the chain. We note that the cluster result is always larger and the discrepancy is quite insensitive to the value of damping. The insensitivity to the value of damping confirms that the discrepancy is not due to the perturbation expansion. To fix this problem, we note that the next set of $(\nu, \mu)$ values discarded by the $(6 \times 6)$ matrix are $(0, \pm 3)(1, \pm 1),(0, \pm 4),(2,0)$, and $(1, \pm 2)$. If these are included, the matrix becomes $(15 \times 15)$. Figure 7 shows the cluster result using $(15 \times 15)$ matrices compared to the slab result. The comparison is almost perfect even at $V_{I}$ $=5.33 \mathrm{eV}$.

In this section, we have shown that for source atoms buried four or more atomic layers deep, the $(6 \times 6)$ separable form may not be enough. However, since the intensity from a buried layer is weak, the error should not be serious for emissions from bulk materials. Higher than $(6 \times 6)$ matrices in the separable form are necessary only if the study is on a buried heterogeneous interface or buried $\delta$-doped layers situated four or more atomic layers below the surface. We have also tested the case of a vertical three-atom $\mathrm{Ni}$ chain separated by $2.49 \AA$ and the emission is from the deepest (third) atom. The results show that the $(6 \times 6)$ separable form is accurate for this case.

\section{Ni(001)-(1×1), CLUSTER SIZE EFFECT}

Having shown that the perturbation approximation in the cluster method diverges for long linear Ni chains with 4-10$\mathrm{eV}$ damping and that the $(6 \times 6)$ separable form causes errors from a few to $100 \%$ in the normalized intensity for four-or-more-layer buried atoms irrespective of damping, we investigate in this section how these factors affect emissions from a solid. We choose a simple test case, a $\mathrm{Ni}(001)$ slab with an ideal $(1 \times 1)$ structure. The initial state is a Ni core level and all elements in Eq. (7) are set to zero except $M_{00}^{\alpha}$ $=1$. This corresponds to an outgoing $s$ wave at the source atom. It is the simplest possible source wave and is, in fact, unphysical because real source waves obey the dipole selection rule. However, the $s$-wave source does provide a minimum standard for testing the approximations used in the cluster approach. Both the slab and cluster calculations use 
identical dynamical inputs: inelastic damping $V_{I}=5.33 \mathrm{eV}$, no temperature correction, the inner potential $V_{0}$ is set to zero, and ten phase shifts are used. The shape of the cluster is chosen as follows: We construct a half ellipsoid, with its midcircle as the surface and its long radius pointing downwards. The radius of the midcircle is $7.8 \AA$ and the long radius is $11.7 \AA$. We then place a $\mathrm{Ni}(001)$ lattice in this half ellipsoid. The surface layer of the Ni lattice is at the midcircle with a $\mathrm{Ni}$ atom at the center of the circle. All Ni atoms in a (001) lattice that fall within the boundaries of the half ellipsoid are included. This construction includes 148 atoms and the atoms lie in seven atomic planes. As indicated before, this is the largest size cluster calculated by the RA method to date. Because of the $A B A B \ldots$ stacking sequence of $\mathrm{Ni}(001)$, the emitting atom is at the center of each odd layer and one of four nearest to center atoms of each even layer. The comparisons with the slab calculations are made separately for emitting atoms in the top, second, and third layers.

Because the cluster approach is a real-space method, the far-field form of the propagator is used in the differential coefficient $d \omega / d \Omega_{\hat{r}}$, without consideration of wave matching at the interface. The ray bending at the interface is then included in an ad hoc manner by using an inside angle: ${ }^{31}$

$$
\theta_{\text {in }}=\tan ^{-1} \frac{\left|k_{f \|}\right|}{\operatorname{Re} k_{\perp \text { in }}}
$$

in the cluster method. We shall see in the next section that this ad hoc model contains significant errors at emission directions glancing to the surface.

The comparisons are shown in Figs. 8-10, with the slab results shown by solid lines and the RA cluster results shown by broken lines. The wave number is from 4 to $10 \AA^{-1}$, corresponding to the $61-381 \mathrm{eV}$ energy range. The detection angles $\theta, \phi$ are measured from the surface normal and [110] direction, respectively. We first comment on the normal emission results. The comparison between slab and cluster results is decent for an emitter in the top or second layer [Figs. 8(a) and 9(a)]. The comparison is unacceptable when the emitter is from the third layer [Fig. 10(a)]. Here, we note major antiphase peaks between $4.5-5.5 \AA^{-1}$ and again between $7-8 \AA^{-1}$. What causes these large discrepancies? For the 148-atom cluster size and lattice geometry, with emitters located in the top three layers of the lattice, the situations of 13-atom-long linear chains and buried source atoms (four layers down) depicted in Figs. 3 and 6 do not exist. Therefore, the discrepancy is not due to MS expansion divergence or the cutoff of the RA separable form. Instead, we attribute the discrepancy as due to insufficient cluster size, bearing in mind for the slab calculation, multiple scattering from many more atoms per layer and many more layers is included. An independent evidence that the discrepancy is not due to perturbation expansion divergence is the fact that increasing the MS order does not alter the cluster result. This shows that the cluster result has converged, except that the converged result is wrong because the cluster size is too small. Because of the shape of the half ellipsoid, increasingly more relevant atoms in the plane are missing from the cluster when the emitter is from a deeper layer. This explains the increasingly poor comparisons for the deeper layer emitters.
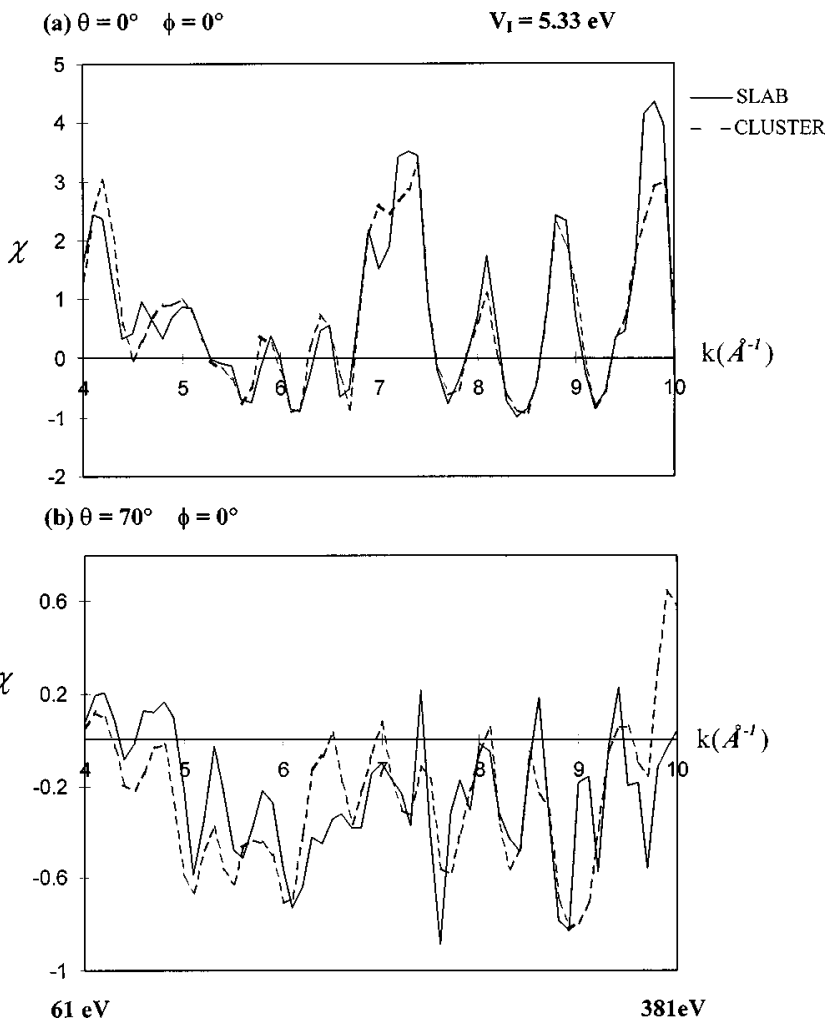

FIG. 8. Comparison of normalized ARPD curves between the slab (solid line) and cluster (broken line) results for $\mathrm{Ni}(001)$ with emitter in the top layer.

A similar trend, i.e., the increasingly poor comparisons with deeper layer emitters, also appears for the glancing angle emission direction, $\theta=70^{\circ}$. However, in addition, there is a sizable worsening in the intensity comparisons.
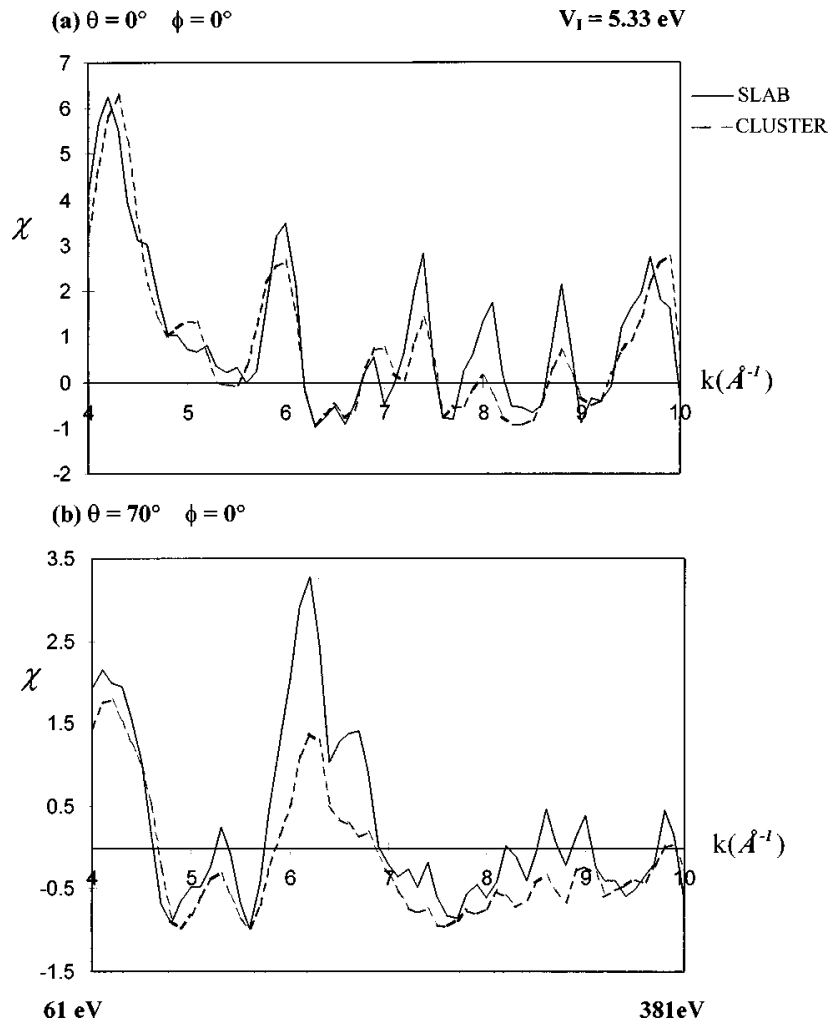

FIG. 9. Same as in Fig. 8, except emitter is in the second layer. 


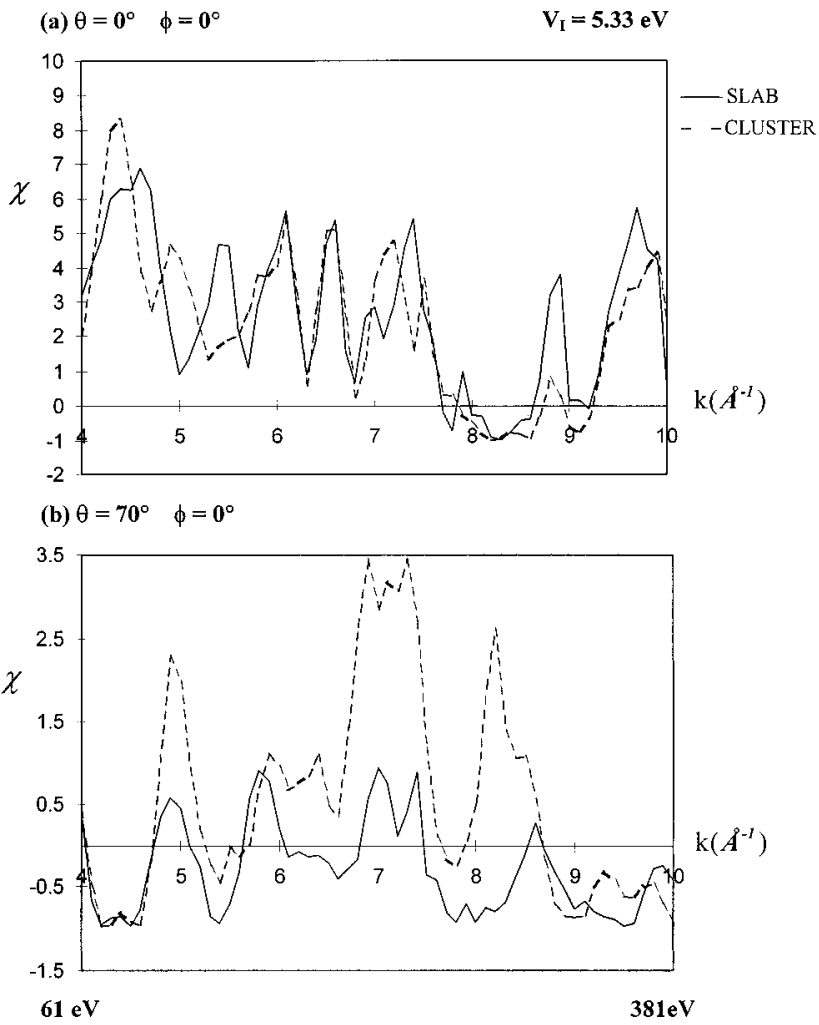

FIG. 10. Same as in Fig. 8, except emitter is in the third layer.

The poor results at $\theta=70^{\circ}$ are due to errors in the boundary condition in the cluster model. This will be discussed in the next section.

\section{THE INTERFACE BOUNDARY CONDITION}

The cluster method sets the last leg of the scattering path at $\mathbf{R}_{\infty}=\hat{k}_{f} R_{\infty}$, which points from the last scattering atom to the detector. Our version of the RA method ${ }^{31}$ makes an $a d$ hoc correction by pointing $\mathbf{R}_{\infty}$ along $\theta_{\text {in }}$ given in Eq. (21). While this adjustment improves the comparison with the slab method, it does not totally correct the effect. The proper way to propagator the photoelectron across an interface, as is done in the slab method, is to start with the wave function at a point $\mathbf{R}$ just inside the interface and transform the nearfield spherical Hankel function into the plane-wave representation. The coefficients of the plane waves with parallel momentum $\mathbf{k}_{f \|}$ are expressed in terms of an expansion in $Y_{l m}\left(\hat{k}_{\text {in }}^{+}\right)$. From Eqs. (2) and (3), we notice that the arguments of the spherical harmonics are complex. Spherical harmonics with complex arguments are defined and used in LEED theory [for the definition of $Y_{l m}(z, \phi)$ with complex $z$, see Ref. 27, Appendix A]. The differential flux $d \omega / d \Omega_{\hat{k}_{f}}$ is then evaluated by finding the number of $\mathbf{k}_{\|}$states the detector collects on the vacuum side. A discussion of this procedure can be found in Ref. 5, Eqs. (1)-(23).

The cluster method, on the other hand, uses spherical harmonics with real $\theta, \phi$ throughout. The method applies the far-field condition from the last scattering site to the detector. This is only justified if the entire space is a single medium (i.e., if the solid's potential $V_{0}+i V_{I}$ fills the entire space, including the detector). In the presence of an interface, the formula used in the cluster method for $d \omega / d \Omega_{\hat{r}}$ is different

\section{Vertical 2-atom Chain}
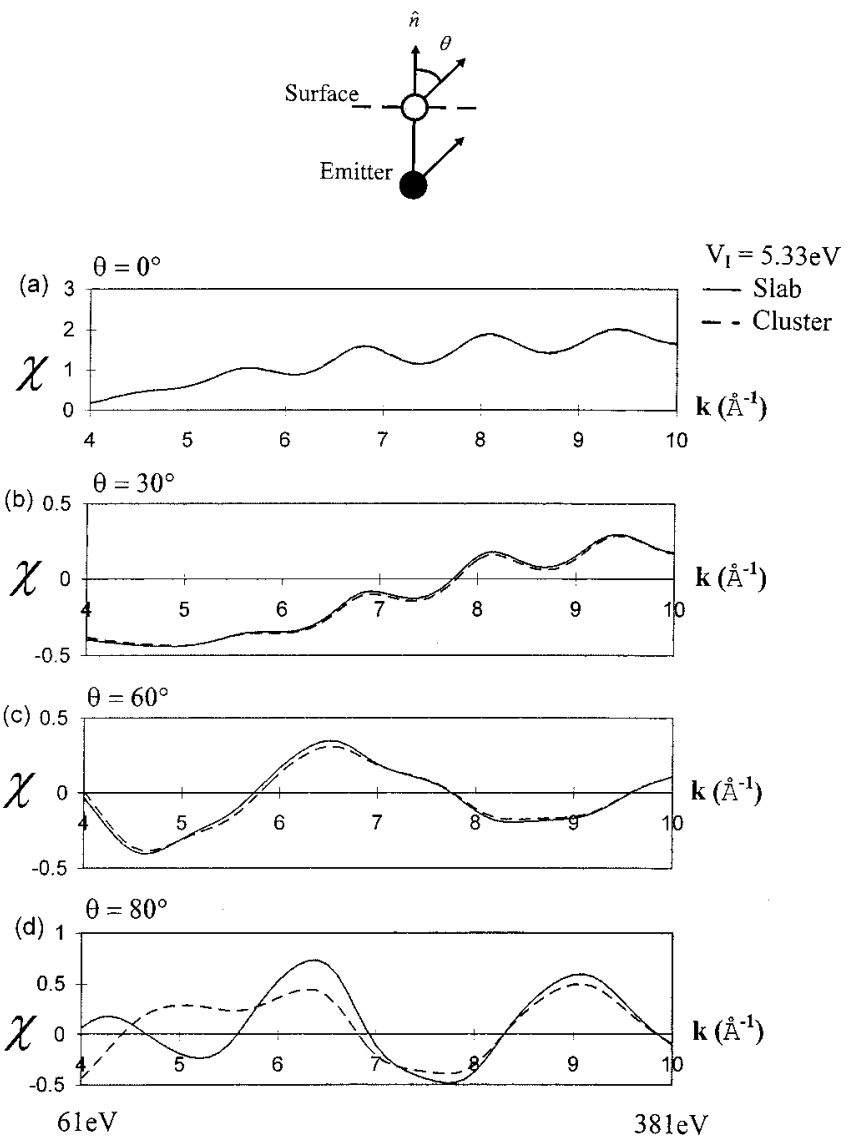

FIG. 11. The slab result (solid line) vs the RA cluster result (dotted line) for a two-atom vertical $\mathrm{Ni}$ chain, with $V_{0}=0 \mathrm{eV}$ and $V_{I}=5.33 \mathrm{eV}$ for various forward scattering angles.

from $d \omega / d \Omega_{\hat{k}_{f}}$. This point has already been discussed in Ref. 5.

To illustrate the effect of the boundary condition, we show in Figs. 11 and 12 the comparison between the cluster and slab methods for a vertical two-Ni atom chain, as a function of the detection angle. Figures 11(a)-11(d) show that the comparison is good for scattering angles varying from $0^{\circ}$ to $60^{\circ}$, but at $\theta=80^{\circ}$, there are large errors in the cluster method, especially at low $k$ values. Figures 12(a)-12(d) exhibit the same trend, with good comparisons at $\theta$ $=130^{\circ}-180^{\circ}$, but the agreement deteriorates at the glancing angle of $100^{\circ}$.

As mentioned in Sec. VI, we have set the inner potential $V_{0}$ to zero in the comparison. For a nonzero $V_{0}$, the error caused by the boundary condition would be even larger. On the other hand, if $V_{0}=0$ and $V_{I} \rightarrow 0$, the two calculations should agree because the solid and vacuum will become the same medium, as is assumed in the cluster boundary condition. We demonstrate this in Figs. 13 and 14 where we have reduced the damping to $1 \mathrm{eV}$ (the inner potential is again set to zero). The improvements at $\theta=80^{\circ}$ and $100^{\circ}$ are large and obvious.

These figures show that the error increases if $V_{0}$ and/or $V_{I}$ increase. Unfortunately, to minimize the boundary condition error, it is necessary to use a very small value of $V_{I}$. However, according to Fig. 3, a very large $V_{I}$ is needed for con- 


\section{Vertical 2-atom Chain}
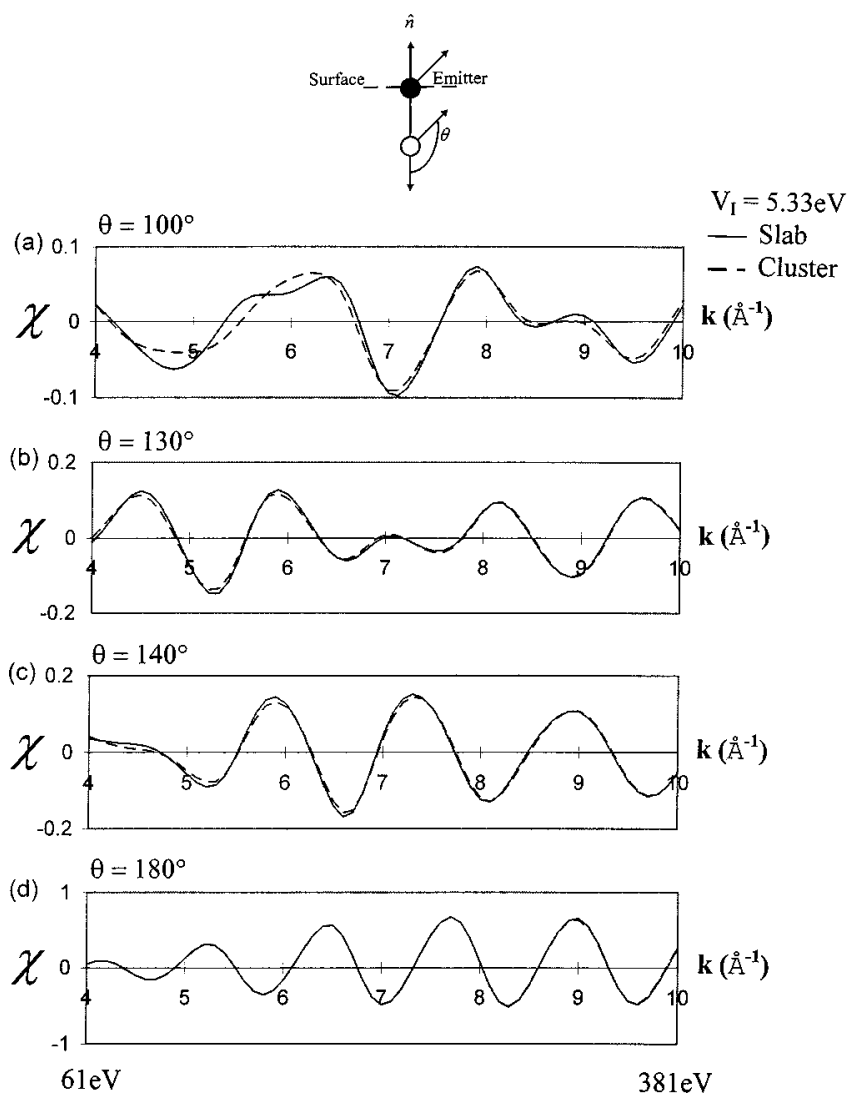

FIG. 12. Same as in Fig. 11, except for various backscattering angles.

vergence of the perturbation expansion approximation. Thus, these two approximations in the cluster method require contradictory inputs to make them work.

The reason why we choose a two-atom chain to test the boundary condition effect is because a two-atom system does not include the separable matrix. Therefore, the truncation problem illustrated in Fig. 6 does not enter here and the discrepancy observed in Figs. 11 and 12 is entirely due to the boundary-condition effect.

\section{CONCLUSIONS AND COMMENTS}

The RA method is originally developed for extended XAFS where it has been shown to work well. ${ }^{23}$ For photoelectron diffraction from ordered systems, the RA method can hardly compete with the slab method for either efficiency or accuracy. The RA method is confined to work within a finite cluster-currently it is difficult to include more than a few hundred atoms. The slab method, on the other hand, has the freedom to use the angular-momentum representation or the plane-wave representation, depending on the scattering strength. In the plane-wave representation, variations in the interlayer distances $\mathbf{d}_{i j}$ are handled very efficiently because these variations only change the exponential coefficients $e^{i \mathbf{k}_{\mathrm{in}}^{ \pm}(\mathbf{g}) \cdot \mathbf{d}_{i j}}$. The layer scattering matrices $T_{\mathbf{g g}^{\prime}}^{ \pm \pm}$and $R_{\mathbf{g g}^{\prime}}^{ \pm \pm}$are unaffected, and hence, many interlayer spacing variations can be calculated in a very short time. The cluster method, on the other hand, works always in real space and one must

\section{Vertical 2-atom Chain}
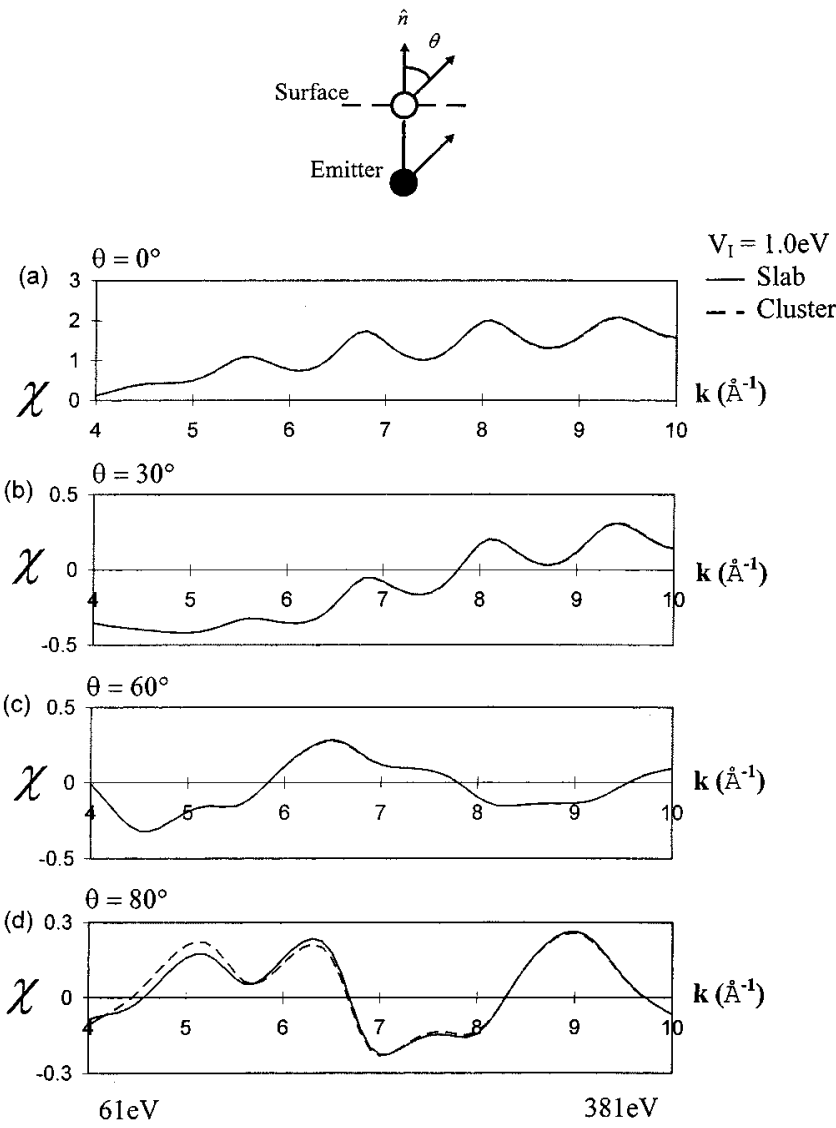

FIG. 13. Same as in Fig. 11, except $V_{0}=0 \mathrm{eV}$ and $V_{I}=1.0 \mathrm{eV}$ are used.

recalculate each new $G_{L, L^{\prime}}\left(k\left|\mathbf{d}_{i}-\mathbf{d}_{j}\right|\right)$ in a complex structural search.

In this paper, we have shown that the truncation of the separable form to $(6 \times 6)$ does not cause serious problems for photoemission from an overlayer or a bulk sample. It would, however, cause intensity errors for emission from a buried (deeper than three layers) heterogeneous interface or $\delta$-doped layers (e.g., errors in the forward focusing ${ }^{32,33}$ intensity). The cluster method's boundary condition causes intensity and peak position errors mainly in small $k$ and large damping situations at grazing collection angles. The most serious problem with the backward summing cluster method is that the perturbation expansion diverges if a cluster is large enough to contain long linear chains. We have recently tested the case of a $\mathrm{Ni}(001)$ plane of atoms with a radius of $12.5 \AA$ (i.e., an 11-atom chain for the diameter and using $V_{I}=4 \mathrm{eV}$ with no temperature correction). The backward summing cluster method diverges with this size of the plane. Unfortunately, planes with smaller radii do not converge to the result given by the slab method.

Because real surfaces have defects and many interface problems involve atomic arrangements with no long-range order, it is important to develop convergent cluster methods. ${ }^{34}$ We now discuss how the backward summing cluster method can be improved. A constraint of the current approach is that before performing a set of calculations, the 


\section{Vertical 2-atom Chain}
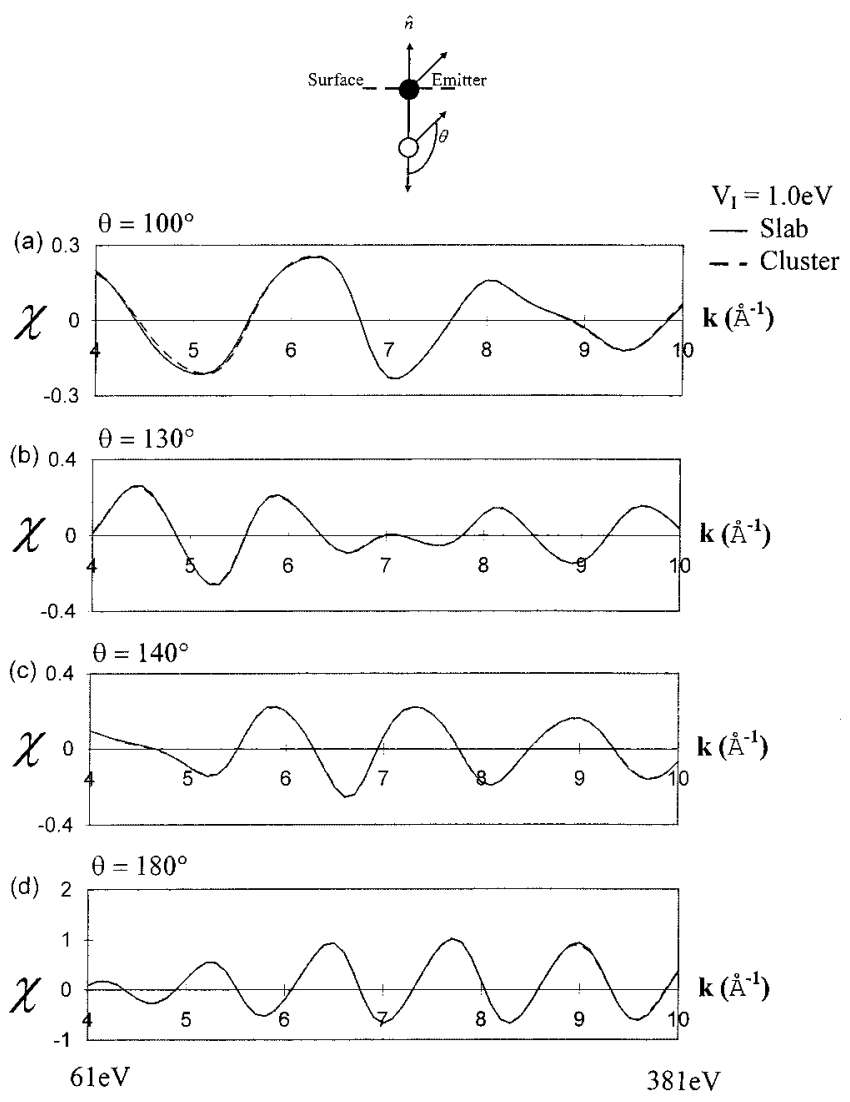

FIG. 14. Same as in Fig. 12, except $V_{0}=0 \mathrm{eV}$ and $V_{I}=1.0 \mathrm{eV}$ are used.

MS order must be preset. If the MS order is set at a large number, an additional parameter called "pathcut" must also be preset. The pathcut tells the computation to discard any amplitude whose value is less than a preset value. In applications where the cluster size is large, the pathcut cannot be too small, in order to save computation time. We have seen that the backward summing cluster method will diverge for some large clusters. If the cluster calculation diverges at some energy, but if the pathcut is preset, the divergence may not always show up as unphysically large numbers. As a result, the cluster calculation may be wrong without the user knowing it. A better approach is to allow the perturbation expansion to increase via iteration. The number of iterations increases until numerical convergence is achieved, if at all.

We have recently reformulated the backward summing cluster method as solving for two-centered scattering amplitudes via a set of simultaneous equations. ${ }^{35}$ We use a relaxation-iteration method to self-consistently solve the set of simultaneous equations to obtain converged two-centered scattering amplitudes. The relaxation method iterates the scattering amplitudes until they converge to within preset accuracies. The number of iterations to achieve convergence depends on structure and dynamical factors such as energy, scattering factors, inelastic damping, etc. The relaxation method extends the realm of convergence for the MS expansion. For example, using the relaxation method, the emission intensity from the plane of $\mathrm{Ni}(001)$ atoms with a $12.5-\AA$ radius $\left(V_{I}=4 \mathrm{eV}\right.$ and with no temperature correction), which is divergent under the backward summing method, becomes convergent. Furthermore, the relaxation method has computation time scaling as $N^{2}$, where $N$ is the dimension of the set of simultaneous equations, instead of $N^{3}$, which is needed in conventional matrix-inversion methods. The relaxation-iteration method is faster than conventional perturbation methods (such as the backward summing method) and at the same time, it produces convergent results in all materials using typical inelastic damping. Details of this method are presented elsewhere. ${ }^{35}$

\section{ACKNOWLEDGMENTS}

This work was supported in part by HK RGC, HK RGC Central Allocation vote, HKU CRCG, U.S. DOE Grant No. DE-FG02-84ER45076, and NSF Grant No. DMR-9214054. We also acknowledge W. P. Lau for his assistance in the preparation of figures for this work.
${ }^{1}$ J. B. Pendry, Low-Energy Electron Diffraction (Academic, London, 1974)

${ }^{2}$ M. A. Van Hove and S. Y. Tong, Surface Crystallography of Low-Energy Electron Diffraction (Springer-Verlag, Heidelberg, 1979).

${ }^{3}$ S. Y. Tong, C. H. Li, and A. R. Lubinsky, Phys. Rev. Lett. 39, 498 (1977); C. H. Li, A. R. Lubinsky, and S. Y. Tong, Phys. Rev. B 17, 3128 (1978).

${ }^{4}$ S. Y. Tong and C. H. Li, in Chemistry and Physics of Solid Surfaces, edited by R. Vanselows and W. England (CRC, Boca Raton, FL, 1982).

${ }^{5}$ S. Y. Tong and H. C. Poon, Phys. Rev. B 37, 2884 (1988).

${ }^{6}$ S. Y. Tong and M. A. Van Hove, Phys. Rev. B 16, 1459 (1977).

${ }^{7}$ C. H. Li and S. Y. Tong, Phys. Rev. Lett. 40, 46 (1978).

${ }^{8}$ S. Y. Tong and C. H. Li, Bull. Am. Phys. Soc. 23, 417 (1978).

${ }^{9}$ S. D. Kevan, D. H. Rosenblatt, D. Denley, B. C. Lu, and D. A. Shirley, Phys. Rev. Lett. 41, 1565 (1978).

${ }^{10}$ D. H. Rosenblatt, J. G. Tobin, M. G. Mason, R. F. David, S. D.
Kevan, D. A. Shirley, C. H. Li, and S. Y. Tong, Phys. Rev. B 23, 3828 (1981); S. D. Kevan, R. F. Davis, J. G. Tobin, D. A. Shirley, C. H. Li, and S. Y. Tong, Phys. Rev. Lett. 46, 629 (1981).

${ }^{11}$ H. Rosenblatt, S. D. Kevan, J. G. Tobin, D. A. Shirley, Y. Huang, and S. Y. Tong, Phys. Rev. B 26, 1812 (1982).

${ }^{12}$ G. Tobin, L. E. Klebanoff, D. H. Rosenblatt, R. F. Davis, E. Umbach, A. G. Baca, D. A. Shirley, Y. Huang, W. M. Kang, and S. Y. Tong, Phys. Rev. B 26, 7076 (1982).

${ }^{13}$ S. Y. Tong, W. M. Kang, D. H. Rosenblatt, J. G. Tobin, and D. A. Shirley, Phys. Rev. B 27, 4632 (1983).

${ }^{14}$ D. H. Rosenblatt, S. D. Kevan, J. G. Tobin, D. A. Shirley, J. C. Tang, and S. Y. Tong, Phys. Rev. B 26, 3181 (1982).

${ }^{15}$ W. M. Kang, C. H. Li, and S. Y. Tong, Phys. Rev. Lett. 47, 931 (1981).

${ }^{16}$ R. A. Bartynski, D. Heskett, K. Garrison, G. Watson, D. M. Zehner, W. N. Mei, S. Y. Tong, and X. Pan, Phys. Rev. B 40, 5340 (1989). 
${ }^{17}$ S. Y. Tong, Hua Li, and H. Huang, Surf. Rev. Lett. 1, 303 (1994).

${ }^{18}$ S. Y. Tong, X. Guo, J. G. Tobin, and G. D. Waddill, Phys. Rev. B 54, 15356 (1996).

${ }^{19}$ G. D. Waddill, J. G. Tobin, X. Guo, and S. Y. Tong, Phys. Rev. B 50, 6774 (1994).

${ }^{20}$ G. D. Waddill, J. G. Tobin, X. Guo, and S. Y. Tong, J. Appl. Phys. 76, 6465 (1994).

${ }^{21}$ J. G. Tobin, E. Tamura, P. A. Sterne, G. D. Waddill, D. P. Pappas, X. Guo, and S. Y. Tong, Spectroscopy 10, 30 (1995).

${ }^{22}$ J. G. Tobin, E. Tamura, P. A. Sterne, G. D. Waddill, D. P. Pappas, X. Guo, and S. Y. Tong, J. Vac. Sci. Technol. A 13, 1534 (1995).

${ }^{23}$ J. J. Rehr and R. C. Albers, Phys. Rev. B 41, 8139 (1990).

${ }^{24}$ J. J. Barton and D. A. Shirley, Phys. Rev. B 32, 1906 (1985).

${ }^{25}$ V. Fritzche, J. Electron Spectrosc. Relat. Phenom. 58, 299 (1992).

${ }^{26}$ A. P. Kaduwela, D. J. Friedman, and C. S. Fadley, J. Electron
Spectrosc. Relat. Phenom. 57, 223 (1991), and references therein.

${ }^{27}$ S. Y. Tong, Surf. Sci. 7, 1 (1975).

${ }^{28}$ W. Moritz, J. Landskron, and T. Greenberg, Surf. Rev. Lett. 4, 469 (1997).

${ }^{29}$ H. Over, W. Moritz, and G. Estl, Phys. Rev. Lett. 70, 315 (1993).

${ }^{30}$ H. Over, M. Gierer, H. Bludau, and G. Estl, Phys. Rev. B 52, 16812 (1995).

${ }^{31}$ Huasheng Wu, Yufeng Chen, D. A. Shirley, and Z. Hussian (unpublished).

${ }^{32}$ H. C. Poon and S. Y. Tong, Phys. Rev. B 30, 6211 (1984).

${ }^{33}$ S. Y. Tong, H. C. Poon, and D. R. Snider, Phys. Rev. B 32, 2096 (1988).

${ }^{34}$ The method by D. K. Saldin, G. R. Harp, and X. Chen, Phys. Rev. B 48, 8234 (1993) is exact, but it requires a large number of partial waves because of the concentric shell treatment.

${ }^{35}$ Huasheng $\mathrm{Wu}$ and $\mathrm{S}$. Y. Tong (unpublished). 